\title{
Existence of solutions converging to zero for nonlinear delayed differential systems
}

\author{
Josef Rebenda ${ }^{1}$ and Zdeněk Šmarda ${ }^{1,2^{*}}$
}

\begin{tabular}{l}
\hline${ }^{*}$ Correspondence: \\
smarda@feec.vutbr.cz \\
${ }^{1}$ CEITEC BUT, Brno University of \\
Technology, Technicka 3058/10, \\
Brno, 61600, Czech Republic \\
2Department of Mathematics, Brno \\
University of Technology, Brno, \\
Czech Republic
\end{tabular}

Czech Republic

\begin{abstract}
We present a result about an interesting asymptotic property of real two-dimensional delayed differential systems satisfying certain sufficient conditions. We employ two previous results, which were obtained using a Razumikhin-type modification of the Ważewski topological method for retarded differential equations and the method of a Lyapunov-Krasovskii functional. The result is illustrated by a nontrivial explanatory example.
\end{abstract}

MSC: 34K12; 34K20

Keywords: differential system with delays; stability of solutions

\section{Introduction}

Various properties of solutions of differential equations with delay were extensively studied recently. Among others we mention [1-9] and the references therein. The results contained in this paper are a generalization of previous research published in [10-12] and [13].

Our aim here is to study the asymptotic behavior of solutions of the following system of differential equations:

$$
x^{\prime}(t)=\mathrm{A}(t) x(t)+\sum_{k=1}^{m} \mathrm{~B}_{k}(t) x\left(\theta_{k}(t)\right)+h\left(t, x(t), x\left(\theta_{1}(t)\right), \ldots, x\left(\theta_{m}(t)\right)\right),
$$

where $t-\theta_{k}(t) \geq 0$ are bounded nonconstant delays satisfying $\lim _{t \rightarrow \infty} \theta_{k}(t)=\infty, \theta_{k}(t)$ are real functions,

$$
h(t, x, y)=\left(h_{1}\left(t, x, y_{1}, \ldots, y_{m}\right), h_{2}\left(t, x, y_{1}, \ldots, y_{m}\right)\right)
$$

is a real vector function, where $x=\left(x_{1}, x_{2}\right), y_{k}=\left(y_{1 k}, y_{2 k}\right)$, and

$$
\mathrm{A}(t)=\left(a_{i j}(t)\right), \quad \mathrm{B}_{k}(t)=\left(b_{i j k}(t)\right), \quad i, j=1,2 ; k=1, \ldots, m,
$$

are real square matrices.

In this paper, we introduce an interesting result, which is a combination of two theorems presented in [14], one regarding the instability of solutions, the other one dealing with the existence of bounded solutions.

(c) 2015 Rebenda and Šmarda. This article is distributed under the terms of the Creative Commons Attribution 4.0 International License (http://creativecommons.org/licenses/by/4.0/), which permits unrestricted use, distribution, and reproduction in any medium, provided you give appropriate credit to the original author(s) and the source, provide a link to the Creative Commons license, and indicate if changes were made. 
It is supposed that the function $h$ satisfies the Carathéodory conditions on $\left[t_{0}, \infty\right) \times$ $\mathbb{R}^{2(m+1)}$, the functions $b_{i j k}$ are locally Lebesgue integrable on $\left[t_{0}, \infty\right)$, and the functions $\theta_{k}$, $a_{i j}$ are locally absolutely continuous on $\left[t_{0}, \infty\right)$.

Since we study two-dimensional systems, we use a transformation into complex variables to simplify the system (1) into one equation with complex coefficients.

The complex variables are defined as $z=x_{1}+i x_{2}, w_{1}=y_{11}+i y_{12}, \ldots, w_{m}=y_{m 1}+i y_{m 2}$. Using this transformation we get

$$
\begin{aligned}
z^{\prime}(t)= & a(t) z(t)+b(t) \bar{z}(t)+\sum_{k=1}^{m}\left[A_{k}(t) z\left(\theta_{k}(t)\right)+B_{k}(t) \bar{z}\left(\theta_{k}(t)\right)\right] \\
& +g\left(t, z(t), z\left(\theta_{1}(t)\right), \ldots, z\left(\theta_{m}(t)\right)\right)
\end{aligned}
$$

where we assume $\left(J=\left[t_{0}, \infty\right)\right)$ :

- $A_{k}, B_{k} \in L_{\mathrm{loc}}(J, \mathbb{C})$ (i.e. locally Lebesgue integrable complex-valued functions on $J$ ) for $k=1, \ldots, m$,

- $\theta_{k} \in A C_{\mathrm{loc}}(J, \mathbb{R})$ (i.e. locally absolutely continuous real-valued functions on $J$ ) for $k=1, \ldots, m$,

- $a, b \in A C_{\text {loc }}(J, \mathbb{C})$ (i.e. locally absolutely continuous complex-valued functions on $J$ ),

- $g \in K\left(J \times \mathbb{C}^{m+1}, \mathbb{C}\right)$ (i.e. a complex-valued function which satisfies the Carathéodory conditions on $\left.J \times \mathbb{C}^{m+1}\right)$.

Obviously, the function $g$ is in general dependent on $\bar{z}$ as well as on every $\bar{z}\left(\theta_{k}\right)$. However, the fact that the function $g$ satisfies the Carathéodory conditions enables us to significantly simplify the notation by using only $z$, since the validity of the Carathéodory conditions is not violated by composing with continuous functions $\bar{z}, \bar{w}_{k}$, and $\theta_{k}$.

The relations between the functions are the following:

$$
\begin{aligned}
& a(t)= \frac{1}{2}\left(a_{11}(t)+a_{22}(t)\right)+\frac{i}{2}\left(a_{21}(t)-a_{12}(t)\right), \\
& b(t)= \frac{1}{2}\left(a_{11}(t)-a_{22}(t)\right)+\frac{i}{2}\left(a_{21}(t)+a_{12}(t)\right), \\
& A_{k}(t)= \frac{1}{2}\left(b_{11 k}(t)+b_{22 k}(t)\right)+\frac{i}{2}\left(b_{21 k}(t)-b_{12 k}(t)\right), \\
& B_{k}(t)= \frac{1}{2}\left(b_{11 k}(t)-b_{22 k}(t)\right)+\frac{i}{2}\left(b_{21 k}(t)+b_{12 k}(t)\right), \\
& g\left(t, z, w_{1}, \ldots, w_{m}\right) \\
&=h_{1}\left(t, \frac{1}{2}(z+\bar{z}), \frac{1}{2 i}(z-\bar{z}), \frac{1}{2}\left(w_{1}+\bar{w}_{1}\right), \ldots, \frac{1}{2 i}\left(w_{m}-\bar{w}_{m}\right)\right) \\
& \quad+i h_{2}\left(t, \frac{1}{2}(z+\bar{z}), \frac{1}{2 i}(z-\bar{z}), \frac{1}{2}\left(w_{1}+\bar{w}_{1}\right), \frac{1}{2 i}\left(w_{1}-\bar{w}_{1}\right), \ldots, \frac{1}{2 i}\left(w_{m}-\bar{w}_{m}\right)\right) .
\end{aligned}
$$

Conversely, putting

$$
\begin{array}{ll}
a_{11}(t)=\operatorname{Re}[a(t)+b(t)], & a_{12}(t)=\operatorname{Im}[b(t)-a(t)], \\
a_{21}(t)=\operatorname{Im}[a(t)+b(t)], & a_{22}(t)=\operatorname{Re}[a(t)-b(t)], \\
b_{11 k}(t)=\operatorname{Re}\left[A_{k}(t)+B_{k}(t)\right], & b_{12 k}(t)=\operatorname{Im}\left[B_{k}(t)-A_{k}(t)\right],
\end{array}
$$




$$
\begin{aligned}
& b_{21 k}(t)=\operatorname{Im}\left[A_{k}(t)+B_{k}(t)\right], \quad b_{22 k}(t)=\operatorname{Re}\left[A_{k}(t)-B_{k}(t)\right], \\
& h_{1}\left(t, x, y_{1}, \ldots, y_{m}\right)=\operatorname{Re} g\left(t, x_{1}+i x_{2}, y_{11}+i y_{12}, \ldots, y_{m 1}+i y_{m 2}\right), \\
& h_{2}\left(t, x, y_{1}, \ldots, y_{m}\right)=\operatorname{Im} g\left(t, x_{1}+i x_{2}, y_{11}+i y_{12}, \ldots, y_{m 1}+i y_{m 2}\right),
\end{aligned}
$$

equation (2) can be written in the real form (1) as well.

The equivalence of the dynamical invariants and asymptotic properties of the solutions of the real system (1) and the complex equation (2) is shown in [15] for the simple case covering ordinary differential equations.

In this paper we consider (2) in the case when

$$
\liminf _{t \rightarrow \infty}(|a(t)|-|b(t)|)>0
$$

and study the behavior of the solutions of (2) under this assumption, which generally means that $\operatorname{det} \mathrm{A}(t)>0$ for $t$ sufficiently large. This situation corresponds to the case when the equilibrium point 0 of the autonomous homogeneous system

$$
x^{\prime}=\mathbf{A} x,
$$

where $\mathbf{A}$ is supposed to be a regular constant matrix, is a center, a focus or a node. Such a situation has some geometrical aspects, which are used in an analysis of the transformed equation (2). See [15] for more details.

Further, we suppose that (2) satisfies the uniqueness property of solutions.

\section{Preliminaries}

Throughout this paper we will assume that

$$
\liminf _{t \rightarrow \infty}(|a(t)|-|b(t)|)>0, \quad t \geq \theta_{k}(t) \geq t-r \text { for } t \geq t_{0}+r
$$

where $r>0$ is a constant, which means that the delays $\theta_{k}$ are bounded. This is the same case as considered in [14]. Similar results for a case different from (5) were obtained in [7].

Then there are numbers $T \geq t_{0}+r$ and $\mu>0$ such that

$$
|a(t)|>|b(t)|+\mu \quad \text { for } t \geq T \text {. }
$$

Denote

$$
c(t)=\frac{\bar{a}(t) b(t)}{|a(t)|}, \quad \gamma(t)=|a(t)|+\sqrt{|a(t)|^{2}-|b(t)|^{2}}
$$

and

$$
\begin{aligned}
& \vartheta(t)=\frac{\operatorname{Re}\left(\gamma(t) \gamma^{\prime}(t)-\bar{c}(t) c^{\prime}(t)\right)-\left|\gamma(t) c^{\prime}(t)-\gamma^{\prime}(t) c(t)\right|}{\gamma^{2}(t)-|c(t)|^{2}}, \\
& \alpha(t)=1-\left|\frac{b(t)}{a(t)}\right| \operatorname{sgn} \operatorname{Re} a(t) .
\end{aligned}
$$

Moreover, we assume the following conditions to be valid: 
(i) The numbers $T \geq t_{0}+r$ and $\mu>0$ satisfy condition (6).

(ii) There are functions $\varrho, \varkappa, \kappa_{k}:[T, \infty) \rightarrow \mathbb{R}$, where $\varrho$ is continuous on $[T, \infty)$, such that

$$
\begin{aligned}
& \left|\gamma(t) g\left(t, z, w_{1}, \ldots, w_{m}\right)+c(t) \bar{g}\left(t, z, w_{1}, \ldots, w_{m}\right)\right| \\
& \quad \leq \varkappa(t)|\gamma(t) z+c(t) \bar{z}|+\sum_{k=1}^{m} \kappa_{k}(t)\left|\gamma\left(\theta_{k}(t)\right) w_{k}+c\left(\theta_{k}(t)\right) \bar{w}_{k}\right|+\varrho(t)
\end{aligned}
$$

for $t \geq T, z, w_{k} \in \mathbb{C}(k=1, \ldots, m)$.

$\left(\mathrm{ii}_{\mathrm{n}}\right)$ There are numbers $R_{n} \geq 0$ and functions $\varkappa_{n}, \kappa_{n k}:[T, \infty) \rightarrow \mathbb{R}$ satisfying the inequality

$$
\begin{aligned}
& \left|\gamma(t) g\left(t, z, w_{1}, \ldots, w_{m}\right)+c(t) \bar{g}\left(t, z, w_{1}, \ldots, w_{m}\right)\right| \\
& \quad \leq \varkappa_{n}(t)|\gamma(t) z+c(t) \bar{z}|+\sum_{k=1}^{m} \kappa_{n k}(t)\left|\gamma\left(\theta_{k}(t)\right) w_{k}+c\left(\theta_{k}(t)\right) \bar{w}_{k}\right|
\end{aligned}
$$

for $t \geq \tau_{n} \geq T,|z|+\sum_{k=1}^{m}\left|w_{k}\right|>R_{n}$.

(iii) The function $\beta \in A C_{\text {loc }}\left([T, \infty), \mathbb{R}_{-}^{0}\right)$ is such that

$$
\theta_{k}^{\prime}(t) \beta(t) \leq-\lambda_{k}(t) \quad \text { a.e. on }[T, \infty)
$$

where $\lambda_{k}$ is given for $t \geq T$ by

$$
\lambda_{k}(t)=\kappa_{k}(t)+\left(\left|A_{k}(t)\right|+\left|B_{k}(t)\right|\right) \frac{\gamma(t)+|c(t)|}{\gamma\left(\theta_{k}(t)\right)-\left|c\left(\theta_{k}(t)\right)\right|} .
$$

(iiii $)$ The function $\beta_{n} \in A C_{\text {loc }}\left([T, \infty), \mathbb{R}_{-}^{0}\right)$ is such that

$$
\theta_{k}^{\prime}(t) \beta_{n}(t) \leq-\lambda_{n k}(t) \quad \text { a.e. on }\left[\tau_{n}, \infty\right)
$$

where $\lambda_{n k}$ is given for $t \geq T$ by

$$
\lambda_{n k}(t)=\kappa_{n k}(t)+\left(\left|A_{k}(t)\right|+\left|B_{k}(t)\right|\right) \frac{\gamma(t)+|c(t)|}{\gamma\left(\theta_{k}(t)\right)-\left|c\left(\theta_{k}(t)\right)\right|} .
$$

(iv $n$ ) The function $\Lambda_{n}$ is real locally Lebesgue integrable and the inequalities $\beta_{n}^{\prime}(t) \geq$ $\Lambda_{n}(t) \beta_{n}(t), \Theta_{n}(t) \geq \Lambda_{n}(t)$ are satisfied for almost all $t \in\left[\tau_{n}, \infty\right)$, where $\Theta_{n}$ is given by $\Theta_{n}(t)=\alpha(t) \operatorname{Re} a(t)+\vartheta(t)-\varkappa_{n}(t)+m \beta_{n}(t)$.

Furthermore, denote

$$
\Theta(t)=\alpha(t) \operatorname{Re} a(t)+\vartheta(t)-\varkappa(t) .
$$

\section{Main results}

First of all, we recall the two results from [14].

Lemma 1 Let the assumptions (i), ( $\left.\mathrm{ii}_{0}\right)$, ( $\left.\mathrm{ii}_{0}\right)$, ( $\left.\mathrm{iv}_{0}\right)$ be fulfilled for some $\tau_{0} \geq T$. Suppose there exist $t_{1} \geq \tau_{0}$ and $v \in(-\infty, \infty)$ such that

$$
\inf _{t \geq t_{1}}\left[\int_{t_{1}}^{t} \Lambda_{0}(s) d s-\ln (\gamma(t)+|c(t)|)\right] \geq v .
$$


If $z(t)$ is any solution of (2) satisfying

$$
\min _{\theta\left(t_{1}\right) \leq s \leq t_{1}}|z(s)|>R_{0}, \quad \Delta\left(t_{1}\right)>R_{0} e^{-v},
$$

where

$$
\begin{aligned}
& \theta(t)=\min _{k=1, \ldots, m} \theta_{k}(t), \\
& \Delta(t)=(\gamma(t)-|c(t)|)|z(t)|+\beta_{0}(t) \max _{\theta(t) \leq s \leq t}|z(s)| \sum_{k=1}^{m} \int_{\theta_{k}(t)}^{t}(\gamma(s)+|c(s)|) d s,
\end{aligned}
$$

then

$$
|z(t)| \geq \frac{\Delta\left(t_{1}\right)}{\gamma(t)+|c(t)|} \exp \left[\int_{t_{1}}^{t} \Lambda_{0}(s) d s\right]
$$

for all $t \geq t_{1}$ for which $z(t)$ is defined.

Lemma 2 Let the conditions (i), (ii), (iii) be fulfilled and $\Lambda, \theta_{k}^{\prime}(k=1, \ldots, m)$ be continuous functions such that inequality $\Lambda(t) \leq \Theta(t)$ holds a.e. on $[T, \infty)$, where $\Theta$ is defined by (13). Suppose that $\xi:[T-r, \infty) \rightarrow \mathbb{R}$ is a continuous function such that

$$
\Lambda(t)+\beta(t) \sum_{k=1}^{m} \theta_{k}^{\prime}(t) \exp \left[-\int_{\theta_{k}(t)}^{t} \xi(s) d s\right]-\xi(t)>\varrho(t) C^{-1} \exp \left[-\int_{T}^{t} \xi(s) d s\right]
$$

for $t \in[T, \infty]$ and some constant $C>0$. Then there exists $a t_{2}>T$ and a solution $z_{0}(t)$ of (2) satisfying

$$
\left|z_{0}(t)\right| \leq \frac{C}{\gamma(t)-|c(t)|} \exp \left[\int_{T}^{t} \xi(s) d s\right]
$$

for $t \geq t_{2}$.

If we combine the previous two results, we are able to prove the following theorem, which is the fundamental result of this paper.

Theorem 1 Assume that the hypotheses (i), (ii), (ii $\left.{ }_{\mathrm{n}}\right)$, (iii), ( $\left.\mathrm{iii}_{\mathrm{n}}\right),\left(\mathrm{iv}_{\mathrm{n}}\right)$ are valid for $T \leq \tau_{n}$, where $0<R_{n}, n \in \mathbb{N}$, $\inf _{n \in \mathbb{N}} R_{n}=0$. Suppose that $\theta_{k}^{\prime}, \Lambda$ are continuous functions such that inequality $\Theta(t) \geq \Lambda(t)$ is satisfied almost everywhere on $[T, \infty)$, where $\Theta(t)=\alpha(t) \operatorname{Re} a(t)+$ $\vartheta(t)-\varkappa(t)$. Let $\xi:[T-r, \infty) \rightarrow \mathbb{R}$ be a continuous function satisfying the inequality

$$
\varrho(t) C^{-1} \exp \left(-\int_{T}^{t} \xi(s) d s\right)<\Lambda(t)+\beta(t) \sum_{k=1}^{m} \theta_{k}^{\prime}(t) \exp \left[-\int_{\theta_{k}(t)}^{t} \xi(s) d s\right]-\xi(t)
$$

for some constant $C>0$ and $t \in[T, \infty)$. Assume

$$
\inf _{\tau_{n} \leq s \leq t<\infty}\left[\int_{s}^{t} \Lambda_{n}(\sigma) d \sigma-\ln (\gamma(t)+|c(t)|)\right] \geq v
$$




$$
\begin{aligned}
& \limsup _{t \rightarrow \infty}\left[\int_{T}^{t}\left(\Lambda_{n}(s)-\xi(s)\right) d s+\ln \frac{\gamma(t)-|c(t)|}{\gamma(t)+|c(t)|}\right]=\infty, \\
& \lim _{t \rightarrow \infty}\left[\beta_{n}(t) \max _{\theta(t) \leq s \leq t} \frac{\exp \left[\int_{T}^{s} \xi(\sigma) d \sigma\right]}{\gamma(s)-|c(s)|} \sum_{k=1}^{m} \int_{\theta_{k}(t)}^{t}(\gamma(s)+|c(s)|) d s\right]=0,
\end{aligned}
$$

for $n \in \mathbb{N}$, where $v \in(-\infty, \infty)$ and $\theta(t)=\min _{k=1, \ldots, m} \theta_{k}(t)$. Then there is a solution $z_{0}(t)$ of (2) with the property

$$
\lim _{t \rightarrow \infty} \min _{\theta(t) \leq s \leq t}\left|z_{0}(s)\right|=0 .
$$

Proof Using Lemma 2 we obtain the existence of $T \leq t_{2}$ and a solution $z_{0}(t)$ of (2) satisfying for $t \geq t_{2}$ the inequality

$$
\left|z_{0}(t)\right| \leq \frac{C}{\gamma(t)-|c(t)|} \exp \left[\int_{T}^{t} \xi(s) d s\right]
$$

From (20) we get

$$
\inf _{\tau \leq t<\infty}\left[\int_{\tau}^{t} \Lambda_{N}(s) d s-\ln (\gamma(t)+|c(t)|)\right] \geq v>-\infty
$$

Lemma 1 yields

$$
\frac{\Psi(\tau)}{\gamma(t)+|c(t)|} \exp \left[\int_{\tau}^{t} \Lambda_{N}(s) d s\right] \leq\left|z_{0}(t)\right|
$$

for $\tau \leq t$, where $\Psi$ is given by

$$
\Psi(\tau)=(\gamma(\tau)-|c(\tau)|)\left|z_{0}(\tau)\right|+\beta_{N}(\tau) \max _{\theta(\tau) \leq s \leq \tau}\left|z_{0}(s)\right| \sum_{k=1}^{m} \int_{\theta_{k}(\tau)}^{\tau}(\gamma(s)+|c(s)|) d s .
$$

Assume that (23) does not hold. This implies the existence of $\varepsilon_{0}>0$ satisfying $\lim \sup _{t \rightarrow \infty} \min _{\theta(t) \leq s \leq t}\left|z_{0}(s)\right|>\varepsilon_{0}$. We take $N \in \mathbb{N}$ such that $\max \left\{R_{N}, \frac{2}{\mu} R_{N} e^{-v}\right\}<\varepsilon_{0}$. Then

$$
\max \left\{R_{N}, \frac{2}{\mu} R_{N} e^{-\nu}\right\}<\min _{\theta(\tau) \leq s \leq \tau}\left|z_{0}(s)\right|
$$

holds for some $\tau>\max \left\{T, \tau_{N}, t_{2}\right\}$. Taking (22) into account we may assume that

$$
\left|\beta_{N}(\tau)\right| C \max _{\theta(\tau) \leq s \leq \tau} \frac{\exp \left[\int_{T}^{s} \xi(\sigma) d \sigma\right]}{\gamma(s)-|c(s)|} \sum_{k=1}^{m} \int_{\theta_{k}(\tau)}^{\tau}(\gamma(s)+|c(s)|) d s<\frac{1}{2} R_{N} e^{-\nu} .
$$

Hence, with respect to (5), (7), (24), (26), (27), and the nonpositiveness of $\beta_{N}$, we obtain

$$
\begin{gathered}
(\gamma(\tau)-|c(\tau)|)\left|z_{0}(\tau)\right|+\beta_{N}(\tau) \max _{\theta(\tau) \leq s \leq \tau}\left|z_{0}(s)\right| \sum_{k=1}^{m} \int_{\theta_{k}(\tau)}^{\tau}(\gamma(s)+|c(s)|) d s \\
\geq(\gamma(\tau)-|c(\tau)|)\left|z_{0}(\tau)\right|+\beta_{N}(\tau) C \max _{\theta(\tau) \leq s \leq \tau} \frac{\exp \left[\int_{T}^{s} \xi(\sigma) d \sigma\right]}{\gamma(s)-|c(s)|} \\
\quad \times \sum_{k=1}^{m} \int_{\theta_{k}(\tau)}^{\tau}(\gamma(s)+|c(s)|) d s \geq \mu \frac{2}{\mu} R_{N} e^{-\nu}-\frac{1}{2} R_{N} e^{-\nu}>R_{N} e^{-\nu} .
\end{gathered}
$$


The inequalities (24) and (25) give the estimation

$$
\frac{\Psi(\tau)}{\gamma(t)+|c(t)|} \exp \left[\int_{\tau}^{t} \Lambda_{N}(s) d s\right] \leq \frac{C}{\gamma(t)-|c(t)|} \exp \left[\int_{T}^{t} \xi(s) d s\right]
$$

which means that

$$
\int_{T}^{t}\left[\Lambda_{N}(s)-\xi(s)\right] d s+\ln \frac{\gamma(t)-|c(t)|}{\gamma(t)+|c(t)|} \leq \int_{T}^{\tau} \Lambda_{N}(s) d s-\ln \left[C^{-1} \Psi(\tau)\right]
$$

for $\tau \leq t$, which is in contradiction to (21). The proof is complete.

Remark 1 Theorem 1 covers more general situations than Theorem 3 in [7], where the different fundamental assumption $\liminf _{t \rightarrow \infty}(|\operatorname{Im} a(t)|-|b(t)|)>0$ is supposed to hold. Indeed, if we take for example $a(t) \equiv 7+i$ and $b(t) \equiv 2 i$, then condition (5) in this paper is satisfied but the condition $\liminf _{t \rightarrow \infty}(|\operatorname{Im} a(t)|-|b(t)|)>0$ is not valid.

The following nontrivial example was constructed to illustrate an application of the theoretical result presented in Theorem 1 .

Example 1 Consider the two-dimensional system of the nonlinear delayed differential equations

$$
\begin{aligned}
& x_{1}^{\prime}(t)=4 x_{1}(t)+\frac{1}{t^{2}} x_{1}(t)-2 x_{2}(t)+\sum_{k=1}^{m} \frac{1}{4 m} \mathrm{e}^{-2 t} x_{1}\left(t-e^{-k t}\right)+e^{-t}, \\
& x_{2}^{\prime}(t)=4 x_{1}(t)+4 x_{2}(t)+\frac{1}{t^{2}} x_{2}(t)-\sum_{k=1}^{m} \frac{1}{4 m} \mathrm{e}^{-2 t} x_{2}\left(t-e^{-k t}\right) .
\end{aligned}
$$

This system can be written in matrix form (1), where

$$
\begin{aligned}
& \mathrm{A}(t)=\left(\begin{array}{cc}
4+\frac{1}{t^{2}} & -2 \\
4 & 4+\frac{1}{t^{2}}
\end{array}\right), \quad \mathrm{B}_{k}(t)=\frac{1}{4 m}\left(\begin{array}{cc}
\mathrm{e}^{-2 t} & 0 \\
0 & \mathrm{e}^{-2 t}
\end{array}\right), \quad \text { and } \\
& h\left(t, x(t), x\left(\theta_{1}(t)\right), \ldots, x\left(\theta_{m}(t)\right)\right)=\left(\begin{array}{c}
\mathrm{e}^{-t} \\
0
\end{array}\right) .
\end{aligned}
$$

Following our approach, we use a transformation into the complex plane and obtain the delayed differential equation (2) with complex-valued coefficients,

$$
z^{\prime}(t)=(4+3 i) z(t)+i \bar{z}(t)+\frac{1}{t^{2}} z(t)+\sum_{k=1}^{m} \frac{1}{4 m} \mathrm{e}^{-2 t} \bar{z}\left(t-e^{-k t}\right)+e^{-t},
$$

where $a(t) \equiv 4+3 i, b(t) \equiv i, A_{k}(t) \equiv 0, B_{k}(t) \equiv 0, \theta_{k}(t)=t-\mathrm{e}^{-k t}$ for $k=1, \ldots, m$, $g\left(t, z, w_{1}, \ldots, w_{m}\right)=\frac{1}{t^{2}} z+\sum_{k=1}^{m} \frac{1}{4 m} \mathrm{e}^{-2 t} w_{k}+\mathrm{e}^{-t}$.

Obviously $t-1 \leq \theta_{k}(t) \leq t$ and $\theta_{k}^{\prime}(t)=1+k \mathrm{e}^{-k t} \geq 1>0$ for $t \geq 0$.

Suppose $t_{0}=0$ and $T \geq 1$. Then $\gamma(t)=|a(t)|+\sqrt{|a(t)|^{2}-|b(t)|^{2}} \equiv 5+2 \sqrt{6}, c(t)=$ $\bar{a}(t) b(t) /|a(t)| \equiv \frac{3+4 i}{5}$. 
Further,

$$
\begin{aligned}
& \left|\gamma(t) g\left(t, z, w_{1}, \ldots, w_{m}\right)+c(t) \bar{g}\left(t, z, w_{1}, \ldots, w_{m}\right)\right| \\
& \quad \leq \frac{\gamma+|c|}{\gamma-|c|} \frac{1}{t^{2}}|\gamma(t) z+c(t) \bar{z}|+\frac{\gamma+|c|}{\gamma-|c|} \sum_{k=1}^{m}\left[\frac{1}{4 m} \mathrm{e}^{-2 t}\left|\gamma\left(\theta_{k}(t)\right) w_{k}+c\left(\theta_{k}(t)\right) \bar{w}_{k}\right|\right]+\mathrm{e}^{-t} \\
& \quad=\frac{\sqrt{3}}{\sqrt{2}} \frac{1}{t^{2}}|\gamma(t) z+c(t) \bar{z}|+\frac{\sqrt{3}}{\sqrt{2}} \frac{\mathrm{e}^{-2 t}}{4 m} \sum_{k=1}^{m}\left|\gamma\left(\theta_{k}(t)\right) w_{k}+c\left(\theta_{k}(t)\right) \bar{w}_{k}\right|+\mathrm{e}^{-t} .
\end{aligned}
$$

Thus conditions (i) and (ii) are fulfilled with $\varkappa(t) \equiv \frac{\sqrt{3}}{t^{2} \sqrt{2}}, \kappa_{k}(t)=\frac{\mathrm{e}^{-2 t} \sqrt{3}}{4 m \sqrt{2}}$, and $\varrho(t)=\mathrm{e}^{-t}$. To meet condition ( $\mathrm{ii}_{\mathrm{n}}$ ), we estimate for $R_{n}=\frac{1}{n}, \tau_{n}=n$, and $|z|>R_{n}$

$$
\begin{aligned}
& \left|\gamma(t) g\left(t, z, w_{1}, \ldots, w_{m}\right)+c(t) \bar{g}\left(t, z, w_{1}, \ldots, w_{m}\right)\right| \\
& \leq \frac{\gamma+|c|}{\gamma-|c|}\left[\frac{1}{t^{2}}+\frac{\varrho(t)}{R_{n}}\right]|\gamma(t) z+c(t) \bar{z}| \\
& \quad+\frac{\gamma+|c|}{\gamma-|c|} \sum_{k=1}^{m}\left[\frac{1}{4 m} \mathrm{e}^{-2 t}\left|\gamma\left(\theta_{k}(t)\right) w_{k}+c\left(\theta_{k}(t)\right) \bar{w}_{k}\right|\right] \\
& =\frac{\sqrt{3}}{\sqrt{2}}\left[\frac{1}{t^{2}}+n \mathrm{e}^{-t}\right]|\gamma(t) z+c(t) \bar{z}|+\frac{\sqrt{3}}{\sqrt{2}} \frac{\mathrm{e}^{-2 t}}{4 m} \sum_{k=1}^{m}\left|\gamma\left(\theta_{k}(t)\right) w_{k}+c\left(\theta_{k}(t)\right) \bar{w}_{k}\right|,
\end{aligned}
$$

where $\varkappa_{n}(t)=\frac{\sqrt{3}}{\sqrt{2}}\left[\frac{1}{t^{2}}+n \mathrm{e}^{-t}\right]$ and $\kappa_{n k}(t)=\frac{\sqrt{3}}{\sqrt{2}} \frac{\mathrm{e}^{-2 t}}{4 m}$.

Condition (iii) holds with

$$
-\lambda_{k}(t)\left(\theta_{k}^{\prime}(t)\right)^{-1}=-\frac{\mathrm{e}^{-2 t} \sqrt{3}}{4 m \sqrt{2}} \frac{1}{1+k e^{-k t}} \geq-\frac{\mathrm{e}^{-2 t} \sqrt{3}}{4 m \sqrt{2}}=\beta(t) .
$$

Condition $\left(\mathrm{iii}_{\mathrm{n}}\right.$ ) is satisfied for

$$
\beta_{n}(t)=-\frac{\mathrm{e}^{-2(t-1)}}{m} \leq-\frac{\mathrm{e}^{-2 t} \sqrt{3}}{4 m \sqrt{2}}=-\lambda_{n k}(t)\left(\theta_{k}^{\prime}(t)\right)^{-1} .
$$

We get condition $\left(i v_{n}\right)$ by setting

$$
\Lambda_{n}(t)=\Theta_{n}(t)=\frac{16}{5}-\frac{\sqrt{3}}{\sqrt{2}}\left[\frac{1}{t^{2}}+n \mathrm{e}^{-t}\right]-\mathrm{e}^{-2(t-1)}>0
$$

Further, we put $\Lambda(t)=\Theta(t)=\frac{16}{5}-\frac{\sqrt{3}}{t^{2} \sqrt{2}}$.

Then condition (19) holds for

$$
\xi(t)=\frac{8}{5}-\frac{\sqrt{3}}{2 t^{2} \sqrt{2}}-\frac{\sqrt{3}}{\sqrt{2}} \frac{\mathrm{e}^{-2 t}}{4 m}-e^{-t}
$$

and $\xi(t)>0$ for $t \geq T=1$.

Now it is not difficult to verify conditions (21) and (20), since $\Lambda_{n}(t)-\xi(t)>0$ and $\Lambda_{n}(t)>$ 0 for $n \in \mathbb{N}$. Investigating the factors of the product in parentheses in (22), we come to the 
conclusion that

$$
\beta_{n}=O\left(\mathrm{e}^{-2 t}\right), \quad \exp \left[\int_{T}^{s} \xi(\sigma) d \sigma\right]=O\left(\mathrm{e}^{1.6 t}\right)
$$

and

$$
\sum_{k=1}^{m} \int_{\theta_{k}(t)}^{t}(\gamma(s)+|c(s)|) d s=O\left(\sum_{k=1}^{m} e^{-k t}\right)=O\left(\mathrm{e}^{-t}\right) .
$$

Consequently, the product of these factors is asymptotically equal to $O\left(\mathrm{e}^{-\delta t}\right)$, where $\delta>0$ and thus condition (22) is satisfied. All assumptions of Theorem 1 are fulfilled and we can conclude that there exist $t_{2}>1$ and a solution $z_{0}(t)$ of (30) satisfying (23) for $t \geq t_{2}$.

Remark 2 This result is slightly surprising. However, it is in good agreement with the well-known fact that introducing delay into an unstable system without delay can cause a change of behavior of the system. Such situations are described and corresponding results are formulated e.g. in [16].

\section{Conclusion}

We proved an interesting result about the stability of two-dimensional systems with bounded delays. Sufficient conditions for the stability of an originally unstable system were presented. The result is in perfect agreement with the results stated and proved in the established literature. An example showed how this result can be used in practice.

Competing interests

The authors declare that they have no competing interests.

\section{Authors' contributions}

The authors have made contributions of the same significance. All authors read and approved the final manuscript.

\section{Acknowledgements}

The work of the authors was realized in CEITEC - Central European Institute of Technology with research infrastructure supported by the project CZ.1.05/1.1.00/02.0068 financed from European Regional Development Fund and the second author was supported by the project FEKT-S-11-2-921 of Faculty of Electrical Engineering and Communication, Brno University of Technology. This support is gratefully acknowledged.

Received: 15 September 2015 Accepted: 5 November 2015 Published online: 14 November 2015

\section{References}

1. Baculíková, B, Džurina, J, Rogovchenko, YV: Oscillation of third order trinomial delay differential equations. Appl. Math. Comput. 218(13), 7023-7033 (2012)

2. Berezansky, L, Braverman, E: On nonoscillation and stability for systems of differential equations with a distributed delay. Automatica 48(4), 612-618 (2012)

3. Berezansky, L, Diblík, J, Svoboda, Z, Šmarda, Z: Simple uniform exponential stability conditions for a system of linear delayed differential equations. Appl. Math. Comput. 250(1), 605-614 (2012)

4. Braverman, E, Berezansky, L: New stability conditions for linear differential equations with several delays. Abstr. Appl. Anal. 2011, Article ID 178568 (2011)

5. Burton, TA: Stability by Fixed Point Theory for Functional Differential Equations, 348 pp. Dover, New York (2006)

6. Olach, R: Positive periodic solutions of delay differential equations. Appl. Math. Lett. 26, 1141-1145 (2013)

7. Šmarda, Z, Rebenda, J: Asymptotic behaviour of a two-dimensional differential system with a finite number of nonconstant delays under the conditions of instability. Abstr. Appl. Anal. 2012, Article ID 952601 (2012)

8. Stević, S: On q-difference asymptotic solutions of a system of nonlinear functional differential equations. Appl. Math. Comput. 219(15), 8295-8301 (2013)

9. Stević, S: Solutions converging to zero of some systems of nonlinear functional differential equations with iterated deviating argument. Appl. Math. Comput. 219(8), 4031-4035 (2012)

10. Kalas, J: Asymptotic behaviour of a two-dimensional differential system with delay under the conditions of instability. Nonlinear Anal. 62, 207-224 (2005) 
11. Kalas, J: Asymptotic properties of an unstable two-dimensional differential system with delay. Math. Bohem. 131, 305-319 (2006)

12. Kalas, J: Asymptotic properties of a two-dimensional differential system with a bounded nonconstant delay under the conditions of instability. Far East J. Math. Sci.: FJMS 29, 513-532 (2008)

13. Kalas, J, Rebenda, J: Asymptotic behaviour of solutions of real two-dimensional differential system with nonconstant delay in an unstable case. Electron. J. Qual. Theory Differ. Equ. 2012, 8 (2012)

14. Kalas, J, Rebenda, J: Asymptotic behaviour of a two-dimensional differential system with a nonconstant delay under the conditions of instability. Math. Bohem. 136(2), 215-224 (2011)

15. Ráb, M, Kalas, J: Stability of dynamical systems in the plane. Differ. Integral Equ. 3, 127-144 (1990)

16. Kolmanovskii, V, Myshkis, A: Introduction to the Theory and Applications of Functional Differential Equations. Kluwer Academic, Dordrecht (1999)

Submit your manuscript to a SpringerOpen ${ }^{\circ}$ journal and benefit from:

$\rightarrow$ Convenient online submission

Rigorous peer review

- Immediate publication on acceptance

- Open access: articles freely available online

- High visibility within the field

- Retaining the copyright to your article

Submit your next manuscript at $\boldsymbol{~ s p r i n g e r o p e n . c o m ~}$ 